\title{
Possible Evidence for Truncated Thin Disks in the Low-Luminosity Active Galactic Nuclei M81 and NGC 4579
}

\section{Citation}

Quataert, Eliot, Tiziana Di Matteo, Ramesh Narayan, and Luis C. Ho. 1999. "Possible Evidence for Truncated Thin Disks in the Low-Luminosity Active Galactic Nuclei M81 and NGC 4579." The Astrophysical Journal 525 (2): L89-92. https://doi.org/10.1086/312353.

\section{Permanent link}

http://nrs.harvard.edu/urn-3:HUL.InstRepos:41384919

\section{Terms of Use}

This article was downloaded from Harvard University's DASH repository, and is made available under the terms and conditions applicable to Other Posted Material, as set forth at http:// nrs.harvard.edu/urn-3:HUL.InstRepos:dash.current.terms-of-use\#LAA

\section{Share Your Story}

The Harvard community has made this article openly available. Please share how this access benefits you. Submit a story. 


\title{
POSSIBLE EVIDENCE FOR TRUNCATED THIN DISKS IN THE LOW-LUMINOSITY ACTIVE GALACTIC NUCLEI M81 AND NGC 4579
}

\author{
Eliot Quataert Tiziana Di Matteo \\ Harvard-Smithsonian Center for Astrophysics, 60 Garden St., Cambridge, MA 02138; equataert, tdimatteo, rnarayan, @cfa.harvard.edu \\ AND \\ Luis C. Ho \\ Carnegie Observatories, 813 Santa Barbara St. Pasadena, CA 91101-1292; lho@ociw.edu
}

Draft version May 3, 2018

\begin{abstract}
M81 and NGC 4579 are two of the few low-luminosity active galactic nuclei which have an estimated mass for the central black hole, detected hard X-ray emission, and detected optical/UV emission. In contrast to the canonical "big blue bump," both have optical/UV spectra which decrease with increasing frequency in a $\nu L_{\nu}$ plot. Barring significant reddening by dust and/or large errors in the black hole mass estimates, the optical/UV spectra of these systems require that the inner edge of a geometrically thin, optically thick, accretion disk lies at $\sim 100$ Schwarzschild radii. The observed X-ray radiation can be explained by an optically thin, two temperature, advection-dominated accretion flow at smaller radii.
\end{abstract}

Subject Headings: accretion, accretion disks - black holes - galaxies: individual (M81, NGC 4579)

\section{INTRODUCTION}

There are several theoretical models for the structure of matter accreting onto a central black hole, e.g., spherical accretion (Bondi 1952), geometrically thin disks (Shakura \& Sunyaev 1973; see Frank, King, \& Raine 1992 for a review) and their associated coronae (e.g., Haardt \& Maraschi 1991), and advection-dominated accretion flows (ADAFs; Ichimaru 1977; Rees et al. 1982; Narayan \& Yi 1994; Abramowicz et al. 1995; see Narayan, Mahadevan, \& Quataert 1998, and Kato, Fukue, \& Mineshige 1998 for reviews). One of the fundamental questions in accretion theory is to what degree these models are relevant for understanding observations of black holes in galactic nuclei and X-ray binaries.

Lasota et al. (1996) suggested that the supermassive black hole in NGC 4258 and most low-luminosity active galactic nuclei (LLAGN) accrete via an ADAF, with any thin disk restricted to large radii. This is a natural identification since ADAFs can only exist in sub-Eddington systems $\left(L \lesssim 0.01-0.1 L_{\text {edd }}\right)$ and their low radiative efficiency may help explain the observed low luminosities.

Part of the difficulty in observationally testing Lasota et al.'s hypothesis is that many LLAGN have hard X-ray continuum properties very similar to those of luminous Seyferts, namely photon indices $\approx 1.8$ (Serlemitsos, Ptak, \& Yaqoob 1996). Hard X-ray continuum observations are therefore not necessarily the best discriminant among theoretical models; what is required to reproduce the observations is an appropriate Comptonizing medium, which both ADAFs and accretion disk coronae can provide.

One must therefore look to different wavebands to more definitively test theoretical models. For example, infrared and optical/UV observations provide excellent probes of the blackbody emission expected from optically thick, geometrically thin, accretion disks (the "big blue bump"; see, e.g., Koratkar \& Blaes 1999 for a review).

In this Letter we investigate the mode of accretion in LLAGN by comparing theoretical models with the broad band spectra of the nuclei of M81 and NGC 4579. These systems are rough analogues of the well studied maser galaxy NGC 4258.

\section{THE OBSERVATIONS}

The criteria we have used in choosing systems to investigate are the following: (1) There is a mass estimate for the central supermassive black hole; in M81 this is a dynamical estimate from stellar kinematics (Bower et al. 1996) while in NGC 4579 the estimate is based on modeling the BLR (Barth et al. 1996). ${ }^{3}$ (2) There are high resolution spectral observations of the nucleus with VLBI, $H S T$, ground based IR, and $A S C A$, supplemented by ROSAT; references to the original data are given in the compilation by Ho (1999; hereafter H99).

\section{THEORETICAL MODELS}

We measure black hole masses in solar units and accretion rates in Eddington units: $M=m M_{\odot}$ and $\dot{M}=\dot{m} \dot{M}_{e d d}$. We take $\dot{M}_{e d d}=10 L_{e d d} / c^{2}=2.2 \times$ $10^{-8} m M_{\odot} \mathrm{yr}^{-1}$, i.e., with a canonical $10 \%$ efficiency. We measure radii in the flow in Schwarzschild units: $R=r R_{s}$, where $R_{s}=2 G M / c^{2}$.

We model emission from a thin accretion disk as a multicolor blackbody (e.g. Frank et al. 1992). The emission is independent of the microphysics of the disk and depends

\footnotetext{
${ }^{1}$ Chandra Fellow; present address: Institute for Advanced Study, School of Natural Sciences, Olden Lane, Princeton, NJ 08540; eliot@ias.edu ${ }^{2}$ Chandra Fellow

${ }^{3}$ Note that this technique worked quite well for M81 (Ho, Filippenko, \& Sargent 1996).
} 
primarily on $m, \dot{m}$, and the inner edge of the disk $\left(r_{\text {in }}\right){ }^{4}$ We take the outer edge of the disk to be at $r_{\text {out }}=10^{5}$ and the inclination of the disk to the line of sight to be $i=60^{\circ}$, but the choices are not crucial. Multicolor blackbodies are known to be a poor approximation to big blue bumps observed in, e.g., quasars (see the recent review by Koratkar \& Blaes 1999 and references therein). For our purposes, however, they suffice, as we seek only a crude estimate of the presence/absence of a thin disk in particular systems. Corrections due to irradiation of the disk by the X-ray source or electron scattering in the disk atmosphere are insufficient to change our general conclusions.

In models in which the thin disk is truncated at $r_{\text {in }}>3$, we model the accretion on the inside as an ADAF (for related models of black hole X-ray binaries see, e.g., Esin, McClintock, \& Narayan 1997). The outer radius of the ADAF is assumed to be equal to the inner radius of the thin disk, $r_{\text {in }}$, corresponding to a thin disk "evaporating" (see, e.g., Meyer \& Meyer-Hofmeister 1994; Honma 1996) to form an ADAF.

In contrast to a thin disk, the predicted spectrum from an ADAF depends on several microphysics parameters, notably the ratio of gas to magnetic pressure, $\beta$, the viscosity parameter, $\alpha$, and the fraction of the turbulent energy in the plasma which heats the electrons, $\delta$. In this paper, we fix $\alpha=0.1, \beta=10$, and $\delta=0.01$ (for motivation, see Quataert \& Narayan 1999). We adjust $\dot{m}$ to reproduce the observed X-ray flux.

\section{COMPARISON WITH THE DATA}

Figure 1 shows the available data for M81 $\left(m=4 \times 10^{6}\right)$ compiled by H99. There are radio detections, IR limits, an optical/UV detection with HST (the two points at $\approx 3 \times 10^{15} \mathrm{~Hz}$ are due to variability in the source), and an $A S C A$ detection in X-rays.

The dotted lines in Figure 1a show geometrically thin accretion disk models with (from top to bottom) $\dot{m}=$ $10^{-2}, 10^{-3}, 3 \times 10^{-4}$, and $3 \times 10^{-5}$. The disks are assumed to extend all the way to the marginally stable orbit for a Schwarzschild black hole $\left(r_{\mathrm{in}}=3\right)$. None of these models is capable of explaining the HST optical/UV data. In particular, the model with roughly the correct flux level $\left(\dot{m} \approx 3 \times 10^{-4}\right)$ is far too hot while the model which fits the higher frequency $H S T$ data $\left(\dot{m} \approx 3 \times 10^{-5}\right)$ cannot account for the lower frequency $H S T$ data.

The solid line in Figure 1a is a thin disk model with $\dot{m} \approx 10^{-2}$ and $r_{\text {in }} \approx 100$, corresponding to a thin disk "truncated" at about 100 Schwarzschild radii. This model reproduces the lower frequency $H S T$ data fairly well. It does somewhat underproduce the higher frequency data, but this is not a serious difficulty because the Compton emission necessary to account for the X-rays can remove this discrepancy (see Fig. 1b).

Note that for fixed $m, r_{\text {out }}$, and $i$, the thin disk model depends on two parameters, $\dot{m}$ and $r_{\mathrm{in}}$. In turn, the observations provide, roughly speaking, two numbers, the luminosity and the maximum temperature of the disk. ${ }^{5}$
This allows one to fit a unique model to the data and determine, to within a factor of few, the values of $\dot{m}$ and $r_{\text {in }}$.

If the above interpretation of the M81 data is correct, it represents good evidence for a truncated disk in a galactic nucleus. This would have important implications for accretion theory. It is therefore worth emphasizing the assumptions made in our analysis. First, we assume that the black hole mass in M81 is relatively accurately determined to be $m \approx 4 \times 10^{6}$ (see Bower et al. 1996 for the dynamical estimate, which is somewhat uncertain). If the true mass were to be larger by a factor of $\gtrsim 10$, the thin disk emission would be colder $\left(T \propto m^{-1 / 4}\right)$ and would therefore be capable of explaining the observations (we find, e.g., that $m=4 \times 10^{7}, \dot{m}=10^{-5}$, and $r_{\text {in }}=3$ can account for the observed optical/UV spectrum).

The interpretation of the optical/UV data is subject to two additional uncertainties: stellar contamination and dust. The importance of stellar contamination has been estimated from the strength of absorption lines in the optical/UV spectrum. Dust is not, however, so readily accounted for. H99 discusses the possibility that reddening by dust can account for the unusual optical/UV emission in M81, NGC 4579, and a number of other LLAGN. He argues that such an explanation is unlikely, but cannot be fully excluded. In particular, any such dust must have properties different from Galactic or SMC dust; moreover, the presence and/or properties of such dust must be a strong function of the luminosity of the AGN (since luminous AGN do not show optical/UV spectra similar to M81). Until better IR limits are available to constrain reemission by dust, it is unlikely that this question will be fully settled. Throughout this paper we interpret the data assuming negligible reddening.

It is important to note that the interpretation of the optical/UV emission from M81 is not sensitive to uncertainties in our modeling of the thin disk emission. More sophisticated disk models generally have color temperatures which exceed the effective temperature (e.g., Shimura \& Takahara 1995), implying that the emission is hotter than a blackbody, not colder as is needed to explain the observations of M81.

Figure $1 \mathrm{~b}$ shows a model of M81 in which the thin disk is truncated at $r_{\mathrm{in}} \approx 100$, inside of which there is an ADAF ( $\dot{m}$ is $\approx 10^{-2}$ in both). The solid line shows the total emission from this model while the dashed line isolates the ADAF contribution. This combined ADAF + disk model provides a good description of the optical/UV and X-ray data. In particular, the X-ray slope is reproduced fairly well by the ADAF model, without tuning any additional parameters. The X-ray emission from the ADAF is due to Compton scattering of synchrotron photons produced by thermal electrons in the near equipartition magnetic field of the accretion flow. For $r_{\text {in }} \sim 100$ and for typical temperature and density profiles in ADAFs, the soft photons produced by the disk at $r \gtrsim 100$ are negligible compared to those produced by synchrotron emission in the ADAF.

More generally, the X-ray data in M81 can be explained

\footnotetext{
${ }^{4}$ In constructing the disk models, a boundary condition must be applied at $r_{\text {in }}$; we choose the standard zero torque boundary condition for which the disk temperature goes to zero at $r_{\mathrm{in}}$; whether this is correct for $r_{\mathrm{in}} \neq 3$ is unclear. It is, however, the conservative approach, as a number of our conclusions will involve limits on $r_{\text {in }}$. Alternative boundary conditions would give larger limits on $r_{\text {in }}$.

${ }^{5}$ The latter follows because the UV slope in M81 requires the HST data to be on the exponential tail of the disk model (which requires $k T_{\max } \sim h \nu_{\text {min }}$, where $\nu_{\text {min }} \approx 3 \times 10^{14} \mathrm{~Hz}$ is the minimum frequency of the HST observations).
} 
by any appropriate "Compton cloud" inside the truncated disk (as in models for the hard state of galactic black hole candidates; see, e.g., Poutanen, Krolik, \& Ryde 1997). The difference between this and the ADAF model is (1) the source of soft photons for Comptonization is taken to be the disk emission, rather than synchrotron emission and (2) there is no dynamics for the cloud, just a specification of its Thomson optical depth $\tau$ and electron temperature $k T_{e}$. The X-rays can also be produced by a corona on top of a thin disk which extends all the way to $r_{\text {in }}=3$. This highlights the issue raised in the introduction: hard X-ray continuum observations cannot always discriminate between ADAF and coronae models. The optical/UV observations discussed above, while they have their own uncertainties, are necessary for further testing theoretical models.

The observed radio emission from M81 cannot be explained by the models considered here. It is probably synchrotron emission by nonthermal electrons in an outflow/jet (there is good evidence for a jet in M81; Bietenholz et al. 1996; Ho et al. 1999).

Figure 2 shows the available data for NGC $4579(m \approx$ $\left.4 \times 10^{6}\right)$. The broad band spectrum is rather similar to that of M81 (although the data are not as good). There is evidence for a declining optical/UV spectrum (with variability) and a canonical "Seyfert" X-ray photon index of $\approx 1.7$. As in M81, the optical/UV spectrum suggests a truncated disk. The dotted lines in Figure $2 \mathrm{a}$ are for thin disk models with $r_{\text {in }}=3$ and $\dot{m}=3 \times 10^{-2}, 10^{-3}$, and $10^{-4}$, none of which can account for the optical/UV data. The solid line in Figure 2a is a disk model with $\dot{m} \approx 0.03$ and $r_{\text {in }} \approx 100$, which accounts qualitatively for the observations.

Figure $2 \mathrm{~b}$ shows an "ADAF + disk" model of NGC 4579 with $\dot{m} \approx 0.03$ and a transition radius of $r_{\text {in }} \approx 100$. As in M81, this reproduces the data reasonably well, although the higher frequency UV data are somewhat underproduced.

\section{DISCUSSION}

Our principal result is that the optical/UV to X-ray emission detected from the nuclei of M81 and NGC 4579 can be well explained by a model in which an optically thick, geometrically thin accretion disk extends down to $\approx 100$ Schwarzschild radii, inside of which lies an optically thin, two-temperature, ADAF. Such an interpretation is relatively independent of "greybody" effects which lead to deviations of the disk emission from a multicolor blackbody. It does, however, assume that dust in the galactic nuclei of interest is unimportant in modifying the intrinsic optical/UV spectrum of the LLAGN and that current black hole mass estimates are accurate to order of magnitude. These assumptions should be investigated in more detail in future work.

Our models can be tested by future X-ray spectroscopy with the $C X O$ and $X M M$. Detection of an iron line with large relativistic broadening in M81 or NGC 4579 would argue strongly against a truncated thin accretion disk. $A S C A$ observations in fact detect an iron line in these sys- tems, but with a centroid energy of $\approx 6.7 \mathrm{keV}$ (see Ishisaki et al. 1996 for M81 and Terashima et al. 1998 for NGC 4579). The M81 line is broadened by $\sim 10 \%$, while there is no evidence for a broad line in NGC 4579. This high energy emission line is incompatible with the $6.4 \mathrm{keV}$ fluorescent line expected from a canonical thin accretion disk. It is possible that such a line could arise in the transition region between an ADAF and a disk, but it is unclear whether one can simultaneously have the transition region subtend sufficient solid angle with respect to the X-ray emitting plasma and be partially, but not fully, ionized. If so, the line should be broadened by $\approx 10 \%$ (but not by more).

To conclude, we briefly place the present results in the context of other recent work on LLAGN. Gammie, Narayan, \& Blandford (1999) argued that an "ADAF + disk" model gives a reasonable description of the broadband spectrum of NGC 4258; they showed that recent IR observations require that the transition radius between the $\mathrm{ADAF}$ and the disk lies at $\approx 10-100$ Schwarzschild radii. The $\dot{m}$ of their model is $\approx 0.005$ (for $\alpha=0.1$ ). There is thus reasonable consistency between the models of M81 and NGC 4579 presented in this paper and Gammie et al.'s model of NGC 4258: as expected theoretically, the models have comparable values of $r_{\text {in }}$ for comparable $\dot{m}$.

By contrast, Di Matteo et al. (1999ab) constructed ADAF models to explain the broad band spectrum of 6 elliptical galaxies in clusters. They argue that values of $\dot{m} \approx 0.03$ at large radii are needed to explain the spectra of, e.g., M87 and NGC 4696. We have compared thin accretion disk models at this $\dot{m}$ with the observed spectra of M87 and NGC 4696. We find that any thin disk must be truncated at $\gtrsim 10^{4}$ Schwarzschild radii in order not to violate limits on the IR emission from these systems. The limits on $r_{\text {in }}$ in the cluster ellipticals therefore exceed the values estimated in M81, NGC 4579, and NGC 4258 by at least two orders of magnitude.

A plausible explanation for this difference in $r_{\text {in }}$ at comparable $\dot{m}$ is the importance of boundary conditions in determining the mode of accretion (cf Begelman 1986). In the ellipticals, a cooling flow supplies hot, roughly spherically symmetric gas to the accretion flow. The gas remains in this state as an ADAF all the way to the black hole. By contrast, in M81, NGC 4579, and NGC 4258, the interstellar medium feeding the accretion flow may reside primarily in a disk. The accretion can proceed via an ADAF on small scales only after the disk has "evaporated" into an ADAF (by a still poorly understood process).

We thank Eric Agol, Anthony Aguirre, Aaron Barth, Julian Krolik, Aneta Siemiginowska, and Marco Spaans for useful discussions. We acknowledge support from an NSF Graduate Research Fellowship (EQ), NSF Grant AST $9820686(\mathrm{RN})$, and NASA/STScI grants GO-06837.0195A, AR-07527.02-96A, and AR-0831.02-97A (LCH). TDM (EQ) acknowledges support provided by NASA through Chandra Fellowship grant number PF8-10005 (PF9-10008) awarded by the Chandra X-ray Center, which is operated by the Smithsonian Astrophysical Observatory for NASA under contract NAS8-39073. 
Abramowicz, M., Chen, X., Kato, S., Lasota, J.P., \& Regev, O., 1995, ApJ, 438, L37

Barth, A. J. et al., 1996, AJ, 112, 1829

Begelman, M. C., 1986, Nature, 322, 614

Bietenholz, M. F. et al., 1996, ApJ, 457, 604

Bondi, H., 1952, MNRAS, 112, 19

Bower, G. A., Wilson, A.S., Heckman, T. M., \& Richstone, D.O., 1996, in The Physics of LINERs in View of Recent Observations, ed. M. Eracleous et al.. (San Francisco: ASP), 163

Di Matteo, T., Fabian, A. C., Rees, M. J., Carilli, C. L., \& Ivison, R. J., 1999a, MNRAS, 305, 492

Di Matteo, T., Quataert, E., Allen, S Narayan, B., \& Fabian, A. C., 1999b, MNRAS, submitted (astro-ph/9905053)

Esin, A. A., McClintock, J. E., \& Narayan, R., 1997, ApJ, 489, 86 Frank, J., King, A., \& Raine, D., 1992, Accretion Power in Astrophysics (Cambridge Univ. Press)

Gammie, C.F., Narayan, R., \& Blandford, R., 1999, ApJ, 516, 177

Haardt, F. \& Maraschi, L., 1991, ApJ, 380, L51

Ho, L. C., 1999, ApJ, 516, 672 (H99)

Ho, L. C., Filippenko, A. V., \& Sargent, W. L. W., 1996, ApJ, 462, 183

Ho, L. C., Van Dyk, S. D., Ppley, G.G.,Sramek, R. A., \& Weiler,

K. W., 1999, AJ, in press (astro-ph/9905077)

Honma, F. 1996, PASJ, 48, 77
Ichimaru, S. 1977, ApJ, 214, 840

Ishisaki, Y. et al., 1996, PASJ, 48, 237

Kato, S., Fukue, J., Mineshige, S., 1998, Black-Hole Accretion Disks (Japan: Kyoto University Press)

Koratkar, A. \& Blaes, O. 1999, PASP, 111, 1

Lasota, J.-P., Abramowicz, M. A., Chen, X., Krolik, J., Narayan, R.,\& Yi, I., 1996, ApJ, 462, 142

Meyer, F. \& Meyer-Hofmeister, E., 1994, A\&A, 288, 175

Narayan, R., Mahadevan, R., \& Quataert, E., 1998, in The Theory of Black Hole Accretion Discs, eds. M.A. Abramowicz, G. Bjornsson, and IE Pringle (Cambridge: Cambridge University Press) (astro-ph/9803131)

Narayan, R., \& Y1, I., 1994 , ApJ, 428, L13

Poutanen, J., Krolik, J. H. \& Ryde, F. 1997, MNRAS, 292, L21

Quataert, E. \& Narayan, R., 1999, ApJ, 520, 298

Rees, M. J., Begelman, M. C., Blandford, R. D., \& Phinney, E. S., 1982, Nature, 295, 17

Serlemitsos, P., Ptak, A., \& Yaqoob, T., 1996, in The Physics of LINERs in View of Recent Observations, eds. Eracleous, M., Koratkar, A., Leitherer, C., \& Ho, L. C. (San Francisco: ASP), 103

Shakura, N. I., \& Sunyaev, R. A., 1973, A\&A, 24, 337

Shimuar, T. \& Takaraha, F., 1995, ApJ, 445, 780

Terashima, Y. et al., 1998, ApJ, 503, 212 

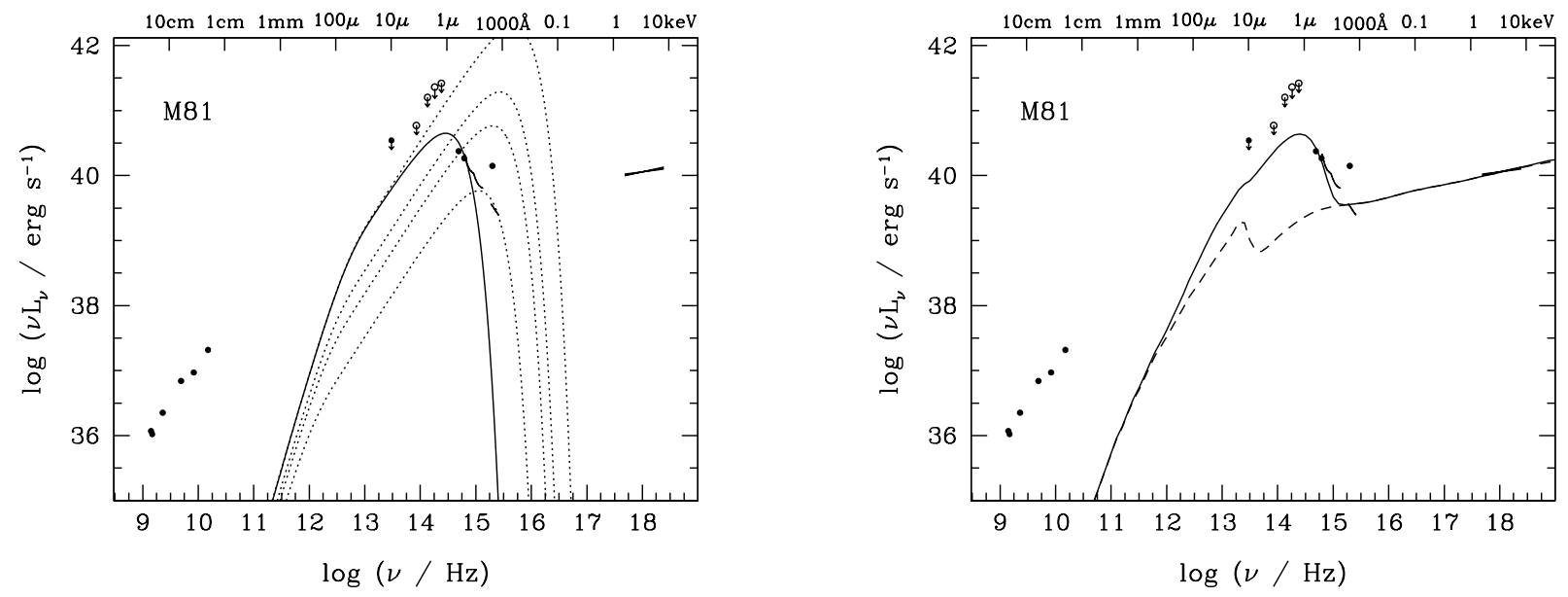

Fig. 1. - Left: Multi-color blackbody thin accretion disk models for the optical-UV emission from M81 (dotted lines from top to bottom: $\dot{m}=10^{-2}, 10^{-3}, 3 \times 10^{-4}$, \& $3 \times 10^{-5}$ with $r_{\text {in }}=3$; solid line: $\left.\dot{m}=3 \times 10^{-3} \& r_{\text {in }}=100\right)$. Right: A model for M81 in which a thin disk is truncated at $r_{\text {in }} \approx 100$, inside of which there is an ADAF. The solid line shows the total "disk + ADAF" emission while the dashed line shows the ADAF contribution. The truncated disk produces the optical/UV emission while the X-rays are produced in the ADAF.
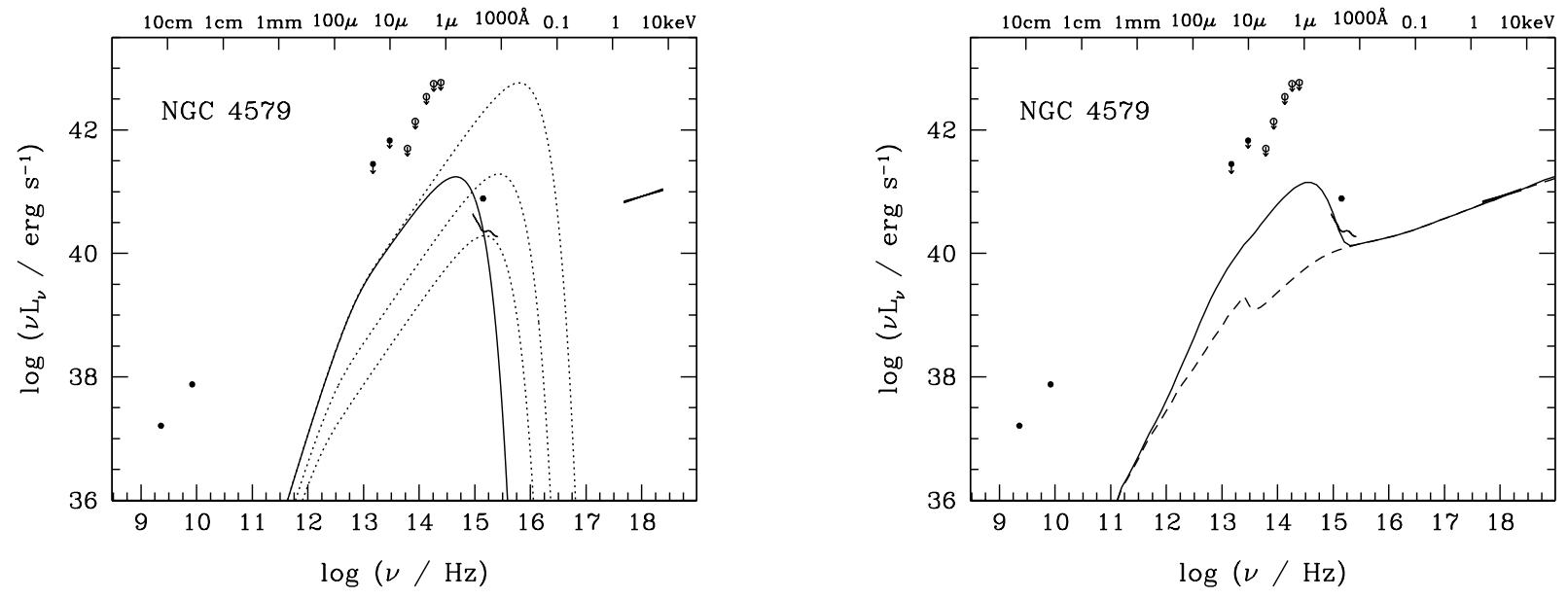

Fig. 2.- Left: Multi-color blackbody thin accretion disk models for the optical-UV emission from NGC 4579 (dotted lines from top to bottom: $\dot{m}=3 \times 10^{-2}, 10^{-3}, \& 10^{-4}$ with $r_{\text {in }}=3$; solid line: $\left.\dot{m}=0.03 \& r_{\text {in }}=100\right)$. Right: A model for NGC 4579 in which a thin disk is truncated at $r_{\text {in }} \approx 100$, inside of which there is an ADAF. The solid line shows the total "disk + ADAF" emission while the dashed line shows the ADAF contribution. 